\section{Complete Resolution of Life-Threatening Bleomycin-Induced Pneumonitis After Treatment With Imatinib Mesylate in a Patient With Hodgkin's Lymphoma: Hope for Severe Chemotherapy-Induced Toxicity?}

\section{Introduction}

Bleomycin-induced pneumonitis (BIP) is a scarcely manageable pulmonary toxicity that occurs in approximately $20 \%$ of patients who are affected with cancers that are treated with bleomycin-containing regimens. Generally, the clinical picture is extremely complicated and therapy is based on steroids. The overall response rate to such therapy is limited. Recent insights in BIP pathogenesis have indicated that a key feature is deregulated mechanisms of tissue repair that are driven by profibrotic cytokines. Here we describe a patient with lifethreatening BIP who was completely cured with imatinib mesylate (IM) after steroids and all other therapies had failed.

\section{Case Report}

A 65-year-old man with stage IV Hodgkin's lymphoma (HL; lymphocyte-depleted variant), already in metabolic complete response after a third cycle of doxorubicin, $50 \mathrm{mg} / \mathrm{m}^{2}$; bleomycin, 10 $\mathrm{U} / \mathrm{m}^{2}$; vinblastine, $6 \mathrm{mg} / \mathrm{m}^{2}$; and dacarbazine, $375 \mathrm{mg} / \mathrm{m}^{2}$, presented with progressive shortness of breath, fever, and dry cough 3 weeks after completion of the sixth cycle of therapy. His medical history was unremarkable. On examination, the patient demonstrated a Karnofsky performance status of $50 \%$, body temperature of $38^{\circ} \mathrm{C}$, blood pressure of 120/70 $\mathrm{mm} \mathrm{Hg}$, pulse rate of 104 beats/min, and respiratory rate of 28 breaths/min. Oxygen saturation was $83 \%$ at room air and $93 \%$ with a $60 \%$ mist mask; arterial blood gases showed $\mathrm{pH}, 7.47$; $\mathrm{PaO}_{2}, 64 \mathrm{~mm} \mathrm{Hg} ; \mathrm{PCO}_{2}, 27 \mathrm{~mm} \mathrm{Hg}$; bicarbonate, $22 \mathrm{mmol} / \mathrm{L}$; and arterial lactate, $2.6 \mathrm{mmol} / \mathrm{L}$. On auscultation, there were crackles and crepitations across the lower and middle lung fields. The WBC was $14,200 / \mu \mathrm{L}$ (reference range, 4,500 to $10,000 / \mu \mathrm{L}$ ) with a normal differ- ential count, hematocrit of $24 \%$, and platelet count of $632,000 / \mu \mathrm{L}$ (reference range, 150,000 to $400,000 / \mu \mathrm{L}$ ). Serum lactic dehydrogenase was $999 \mathrm{UI} / \mu \mathrm{L}$ (reference range $<620 \mathrm{UI} / \mu \mathrm{L}$ ) and the C-reactive protein level was $0.23 \mathrm{~g} / \mathrm{L}$ (reference range $<0.005 \mathrm{~g} / \mathrm{L}$ ). The remaining laboratory results were unremarkable. On admission, a chest radiograph showed bilateral diffuse parenchymal airspace densities with reticular opacities. A high-resolution computed tomography (HRCT) scan of the chest (Fig 1) showed bilateral diffuse ground-glass opacities associated with fine interlobular and intralobular reticulations with honeycomb features. Remarkably, both a CT scan and a CT-positron emission tomography scan confirmed a radiologic and metabolic complete response of HL.

On the basis of this clinical picture, broad-spectrum antibiotic therapy was begun with teicoplanin (initial dose of $400 \mathrm{mg}$ intravenously [IV] every 12 hours and then $800 \mathrm{mg}$ IV once daily) and meropenem ( 1 g every 8 hours) along with fluconazole (200 mg IV every 12 hours) and acyclovir (500 mg IV every 12 hours). After 7 days, HRCT was repeated and showed a slight improvement of the lesions in the upper lobes but no change in the diffuse ground-glass opacities in the lower lobes. Therefore, a bronchoscopy was performed for microbiologic cultures, bronchoalveolar lavage, and cytology evaluation. Pseudomonas aeruginosa was isolated, whereas both bronchoalveolar lavage and cytology were negative for either tumoral cells or other pathogenic microorganisms (including viruses and intracellular bacteria). Because the patient's respiratory condition was progressively deteriorating, treatment with trimethoprim/sulfamethoxazole (4 $\mathrm{mg} / \mathrm{kg}$ every 6 hours) and voriconazole (initial dose of $6 \mathrm{mg} / \mathrm{kg}$ every 12 hours and then $4 \mathrm{mg} / \mathrm{kg}$ every 12 hours) was begun on the recommendation of an infectious diseases consultant.

Given that BIP was suspected, the patient was evaluated to determine whether a lung biopsy should be performed; however, the thoracic surgeon determined that a lung biopsy was contraindicated because of the patient's worsening clinical condition. Therefore, treatment with methylprednisolone $2 \mathrm{mg} / \mathrm{kg}$ was also begun. In addition,

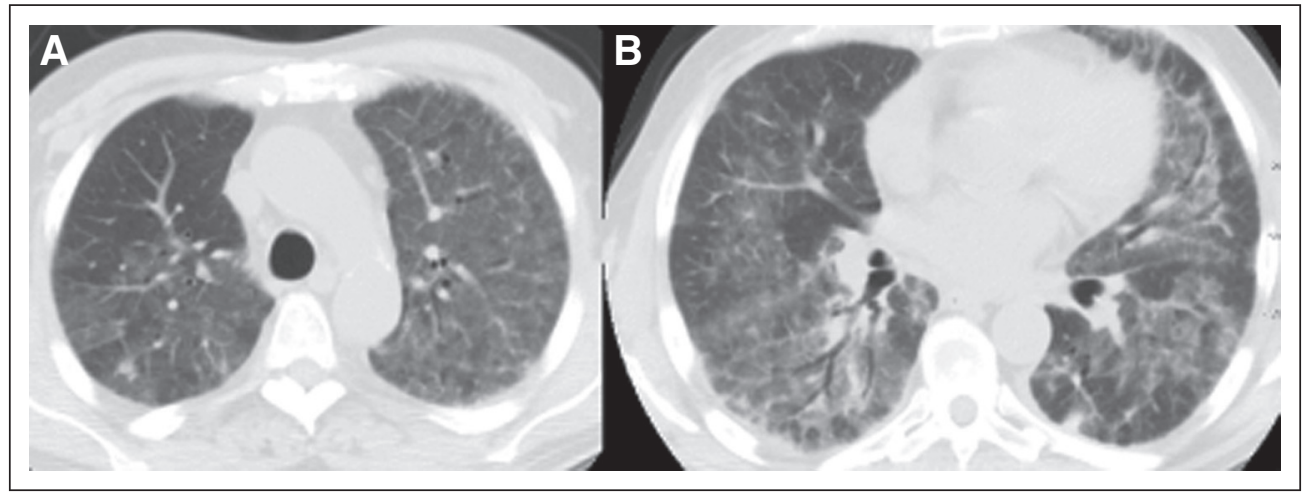

Fig 1. 


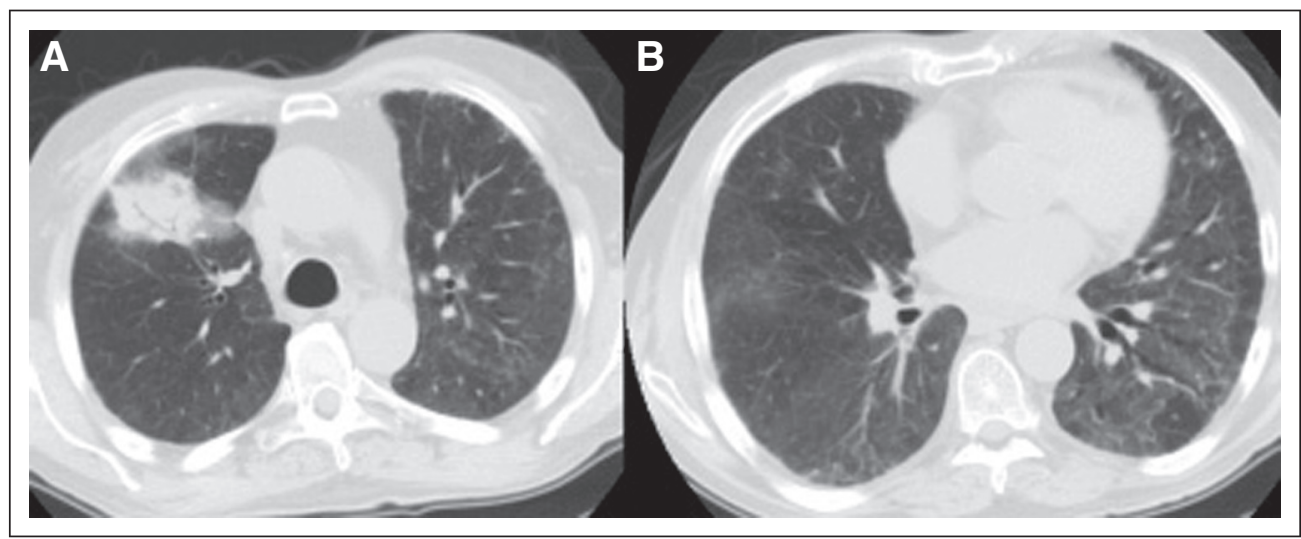

Fig 2.

the patient began receiving continuous positive airway pressure oxygen therapy 72 hours later, but there was no clinical improvement by day 6. In light of this continuous respiratory deterioration and because $\mathrm{BIP}$ was considered to be the most likely diagnosis, after a patient care conference, we began therapy with $300 \mathrm{mg}$ of IM, on the basis of recent data regarding the effectiveness of IM in the setting of allogeneic hematopoietic stem-cell transplantation for the treatment of graftversus-host-induced lung fibrosis.

After a week of IM therapy, the clinical picture began to improve, allowing a reduction in oxygen therapy and then complete suspension of oxygen therapy 21 days after the start of treatment with IM. IM was continued for 6 months and resulted in additional improvement in respiratory function. HRCT scans performed at 3 and 6 months (Figs 2 and 3, respectively) showed a continuous improvement of the interstitial lesions until they completely disappeared. Notably, at 3 months the patient was started on moxifloxacin $400 \mathrm{mg}$ once daily for the onset of asymptomatic focal pneumonia (Fig 2). At the most recent follow-up, the patient is alive and is still in complete remission for HL, with a Karnofsky performance status of 100\%.

\section{Discussion}

Bleomycin-induced lung fibrosis is a major pulmonary toxicity that occurs in approximately $20 \%$ to $40 \%$ of patients who are affected by Hodgkin's lymphoma (HL) and are treated with a bleomycincontaining regimen. ${ }^{1,2}$ The mortality of this complication is high, ranging from $10 \%$ to $20 \%$, and significantly impacts quality of life and 5 -year overall survival. ${ }^{3}$ The BIP clinical picture is usually characterized by dyspnea after exertion, persistent nonproductive cough, and mild fever. At physical examination, findings may be not specific and range from tachypnea only to dry crackles. Generally, the possibility of BIP is suggested by chest imaging studies. The presence of respiratory symptoms and bilateral interstitial infiltrates on CT scans along with the exclusion of active lung infections (defined as negative cultures and lack of response to antimicrobials) are the most important clues to clinical diagnosis. Whenever feasible, the diagnosis relies on a lung biopsy that demonstrates a heterogeneous appearance with alternating areas of normal lung, interstitial inflammation, foci of proliferating fibroblast, dense collagen fibrosis, and honeycomb changes. ${ }^{4}$

BIP pathogenesis is unknown, but there is consensus that deregulated mechanisms of tissue repair driven by profibrotic cytokines initiate the lung-damaging effects. ${ }^{5,6}$ Specifically, tumor growth factor $\beta$, platelet-derived growth factor receptor $\alpha$ (PDGFR- $\alpha$ ), and tumor necrosis factor $\alpha$ are believed to cause transformation, proliferation, and accumulation of fibroblasts with the deposition of an extracellular matrix. ${ }^{7}$ The progressive accumulation of this collagen matrix determines distortion and destruction of the alveolar structures and, eventually, loss of lung function. In animal models, it has been demonstrated that PDGF- $\alpha$ expression is increased in BIP. Finally,

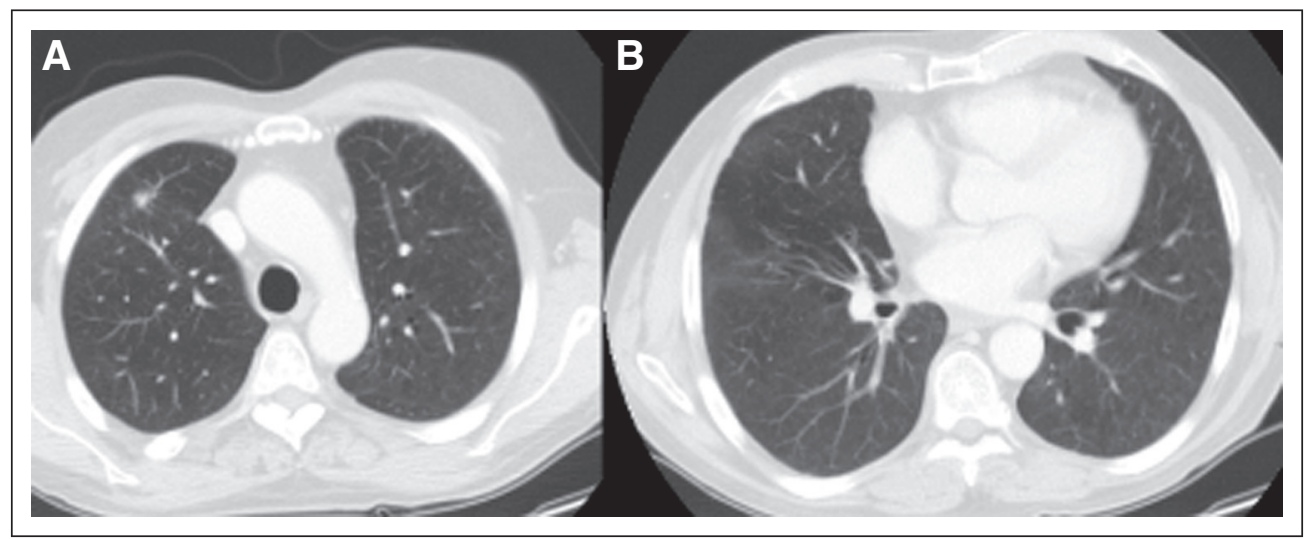

Fig 3. 
enhanced expression of PDGFR- $\alpha$ was also shown in the epithelial cells and alveolar macrophages in lungs of patients with idiopathic pulmonary fibrosis.

Imatinib mesylate is a potent and specific receptor tyrosine kinase inhibitor of ABL, BCR-ABL, KIT, and both isoforms of PDGFR. Since its first use in chronic myeloid leukemia, IM was proven to be active in various PDGFR-driven diseases such as GI stromal tumors, dermatofibrosarcoma protuberans, and chordoma. ${ }^{8,9}$ The successful inhibition of PDGFR that was observed in these tumors suggested that scleroderma-like chronic graft-versus-host disease, a serious adverse event of allogeneic stem-cell transplantation, might be treatable with IM. ${ }^{10}$ Indeed, it is well known that both PDGFR and tumor growth factor $\beta$ pathways are actively involved in the pathogenesis of this complication. The same receptors are involved in idiopathic pulmonary fibrosis, which is a pathologic condition that closely resembles the final lung damage observed in BIP. ${ }^{11}$ These speculations prompted us to test a low-toxicity profile drug such as IM in a patient whose rapidly worsening clinical condition had been refractory to all previously attempted therapies.

As described in the case of this patient, the diagnosis of interstitial lung diseases and BIP in particular is challenging and often depends on clinical, radiologic, and cytologic findings, as well as on the absence of response to all antibiotics used. The extremely favorable clinical outcome of our patient is noteworthy, and the observed complete response warrants additional study of IM in the treatment of a scarcely manageable chronic adverse effect of HL and testicular cancer treatments.

Fabrizio Carnevale-Schianca, Susanna Gallo, Delia Rota-Scalabrini, Dario Sangiolo, Marco Fizzotti, Daniela Caravelli, Antonio Capaldi, Giancarlo Anselmetti, Erica Palesandro, Lorenzo D’Ambrosio, Valentina Coha,
Remo Obert, Massimo Aglietta, and Giovanni Grignani

Institute for Cancer Research and Treatment, Candiolo, Turin, Italy

AUTHORS' DISCLOSURES OF POTENTIAL CONFLICTS OF INTEREST

The author(s) indicated no potential conflicts of interest.

\section{REFERENCES}

1. Martin WG, Ristow KM, Habermann TM, et al: Bleomycin pulmonary toxicity has a negative impact on the outcome of patients with Hodgkin's lymphoma. J Clin Oncol 23:7614-7620, 2005

2. Sleijfer S: Bleomycin-induced pneumonitis. Chest 120:617-624, 2001

3. Lewis BM, Izbicki R: Routine pulmonary function tests during bleomycin therapy: Tests may be ineffective and potentially misleading. JAMA 243:347-351, 1980

4. American Thoracic Society: Idiopathic pulmonary fibrosis: Diagnosis and treatment-International consensus statement. American Thoracic Society (ATS), and the European Respiratory Society (ERS). Am J Respir Crit Care Med 161:646-664, 2000

5. Sime PJ, O'Reilly KM: Fibrosis of the lung and other tissues: New concepts in pathogenesis and treatment. Clin Immunol 99:308-319, 2001

6. Strieter RM: Mechanisms of pulmonary fibrosis: Conference summary. Chest 120:77S-85S, 2001

7. Yoshida M, Sakuma J, Hayashi S, et al: A histologically distinctive interstitial pneumonia induced by overexpression of the interleukin 6 , transforming growth factor beta 1, or platelet-derived growth factor B gene. Proc Natl Acad Sci U S A 92:9570-9574, 1995

8. Druker BJ, Talpaz M, Resta DJ, et al: Efficacy and safety of a specific inhibitor of the BCR-ABL tyrosine kinase in chronic myeloid leukemia. N Engl J Med 344:1031-1037, 2001

9. Demetri GD, von Mehren M, Blanke CD, et al: Efficacy and safety of imatinib mesylate in advanced gastrointestinal stromal tumors. N Engl J Med 347:472-480, 2002

10. Olivieri $A$, Locatelli $F$, Zecca $M$, et al: Imatinib for refractory chronic graft-versus-host disease with fibrotic features. Blood 114:709-718, 2009

11. Daniels $C E$, Wilkes $M C$, Edens $M$, et al: Imatinib mesylate inhibits the profibrogenic activity of TGF-beta and prevents bleomycin-mediated lung fibrosis. J Clin Invest 114:1308-1316, 2004

DOI: 10.1200/JCO.2011.35.6733; published online ahead of print at www.jco.org on June 27, 2011 